\title{
Les manuscrits de Jane Austen : sur la page et dans la durée
}

\section{Kathryn Sutherland}

Traducteur : Nathalie Ferrand

\section{OpenEdition \\ Journals}

Édition électronique

URL : http://journals.openedition.org/genesis/1563

DOI : 10.4000/genesis. 1563

ISSN : 2268-1590

Éditeur :

Presses universitaires de Paris Sorbonne (PUPS), Société internationale de génétique artistique littéraire et scientifique (SIGALES)

\section{Édition imprimée}

Date de publication : 27 novembre 2015

Pagination : 187-202

ISBN : 9791023105049

ISSN : 1167-5101

Référence électronique

Kathryn Sutherland, « Les manuscrits de Jane Austen : sur la page et dans la durée », Genesis [En ligne], 41 | 2015, mis en ligne le 04 mai 2017, consulté le 16 mai 2019. URL : http:// journals.openedition.org/genesis/1563; DOI : 10.4000/genesis.1563 


\title{
Les manuscrits de Jane Austen : sur la page et dans la durée
}

\author{
Kathryn Sutherland
}

Le terme «manuscrit » est communément utilisé, en anglais et en français, pour désigner deux types de réalité : scripturale et matérielle. Car les manuscrits sont à la fois des inscriptions écrites (des textes) et leurs supports (des objets) ; et lorsque nous parlons d'eux, nous nous référons à ces deux dimensions : le manuscrit littéraire et le manuscrit matériel ${ }^{1}$. Les éditeurs scientifiques négligent parfois la relation qui les unit, mais ce n'est certainement pas le cas de l'écrivain qui compose ou transcrit. À eux seuls les mots sur la page n'offrent qu'un aperçu incomplet des processus de la création; en tant que supports physiques du texte écrit, les manuscrits fournissent des indices sur la manière dont un auteur travaille. Ensemble le texte et l'objet présentent un faisceau d'indices et de significations et l'interaction de circonstances internes et externes par lesquelles les mécanismes de l'esprit écrivant sont détectables à travers leur support d'écriture, en même temps qu'ils sont structurés par et contre lui. De là la valeur d'une approche documentaire de l'édition des manuscrits de travail, une approche qui reconnaisse l'association des dimensions matérielle et scripturale et l'importance de la mise en page pour le sens.

Dans le cas des fictions de Jane Austen, les manuscrits qui ont survécu jusqu'à aujourd'hui appartiennent à deux catégories : il s'agit d'une part de brouillons de travail inachevés, abandonnés ou rejetés, d'autre part de mises au net intactes ou portant très peu de corrections, qui ont aussi pour particularité d'être des publications confidentielles ${ }^{2}$ c'est-à-dire que leur aspect formel ou informel (la présence de dédicaces ou de divers éléments paratextuels, ainsi que l'intrusion de mains autres que celle de l'auteur) révèle que ces copies au propre étaient destinées et délibérément confectionnées pour une circulation privée, dans le cercle familial et amical. Les manuscrits de jeunesse-Volume the First, Volume the Second et Volume the Third - et la nouvelle connue sous le titre de Lady Susan, présentent tous les caractéristiques d'une publication confidentielle. En revanche, rien ne prouve qu'Austen considérait la masse des manuscrits de fiction plus tardifs autrement que comme provisoire : des états préparatoires avant publication portés à différents degrés de développement. Entre dans cette dernière catégorie une portion non négligeable d'un roman ou nouvelle abandonnée, intitulée The Watsons, qu'on peut dater d'après son papier et diverses informations biographiques autour de 1803-1805 ; les deux chapitres éliminés de Persuasion datés de la main d'Austen de juillet 1816 ; et douze chapitres de Sanditon composés, d'après une data-

1. «Un problème qui a rapidement surgi était de savoir ce que nous devions considérer comme premier dans notre classification, le manuscrit en tant qu'écriture ou en tant qu'objet de papier ? », dans Kenneth Neil Cameron (dir.), Shelley and his Circle, 1773-1822, vol. I, Cambridge Mass./London, Harvard University Press/Oxford University Press, 1961, « Editorial Procedures », p. XI

2. Je me fonde ici sur la distinction avancée par Donald Reiman quand il affirme que l'intention de l'auteur quant au fait qu'un manuscrit soit privé, public ou confidentiel est essentielle pour comprendre ce que dit ce manuscrit. Une telle information peut relever du non-écrit : ses contextes de genèse et de réception. Les «publications confidentielles » sont les manuscrits « adressés à un groupe particulier de personnes, qui soit sont toutes connues personnellement par l'écrivain, soit appartiennent à un groupe prédéfini dont l'auteur a raison de supposer qu'il partage des valeurs communes avec lui », The Study of Modern Manuscripts: Public, Confidential, and Private, Baltimore/London, Johns Hopkins University Press, 1993, p. 39. 
tion interne, entre le 27 janvier et le 18 mars 1817 . La distinction entre transcription et rédaction originelle se dégage de l'aspect du manuscrit : les mises au net ou les publications confidentielles se trouvent dans des cahiers achetés dans le commerce ; les brouillons dans des cahiers pliés assemblés ou des livrets confectionnés à la maison, dont les structures informelles suggèrent une relation de possession plus étroite et exclusive de l'auteur avec ses textes ${ }^{3}$.

De quelle manière les caractéristiques matérielles des manuscrits (les informations tirées de la structure des cahiers, de la nature du papier, son format et sa fabrication, de l'encre, de l'orthographe et de la ponctuation, de la disposition des mots sur la page) peuvent-elles contribuer à une compréhension de la signification et des finalités d'un texte ? Que disent les structures du manuscrit de la manière qu'avait Austen de préparer ses matériaux ? de ses habitudes de rédaction et de révision ? de son anticipation ou de ses attentes concernant leur réception?

\section{Les cahiers achetés dans le commerce (Notebooks)}

Les trois cahiers de jeunesse, connus d'après les inscriptions portées sur la page de couverture comme Volume the First, Volume the Second et Volume the Third, constituent la plus grande partie des manuscrits confidentiels (fig. 1). Ils représentent les écrits les plus anciens d'Austen (un mélange de nouvelles, pièces, poésies, récits parodiques et essais moraux), écrits et recopiés entre 12 et 18 ans (1787-1793), mais avec des ajouts tardifs qui peuvent aller jusqu'à l'année 1814, lorsqu'elle a 38 ans. Ces écrits de jeunesse sont longtemps apparus aux yeux des critiques comme des objets finis, des tentatives de fabrication de livres dont les structures imitent ou parodient les conventions bibliographiques. Les cahiers reliés du commerce confèrent à leur contenu une forme d'autorité incarnée dans la signature de «The Author », fièrement exhibée dans treize morceaux du Volume the First (fig. 2). Les cahiers semblent être une archive, destinée à servir de référence et de vitrine, des dernières versions d'œuvres brèves qui peuvent avoir mené différentes vies et servi divers buts avant leur transcription sous cette forme et dans cet espace. Les cahiers gardent la mémoire des incursions qu'Austen avait tentées avec succès dans diverses formes de pratiques littéraires de la sociabilité - pièces, récits, poésie.

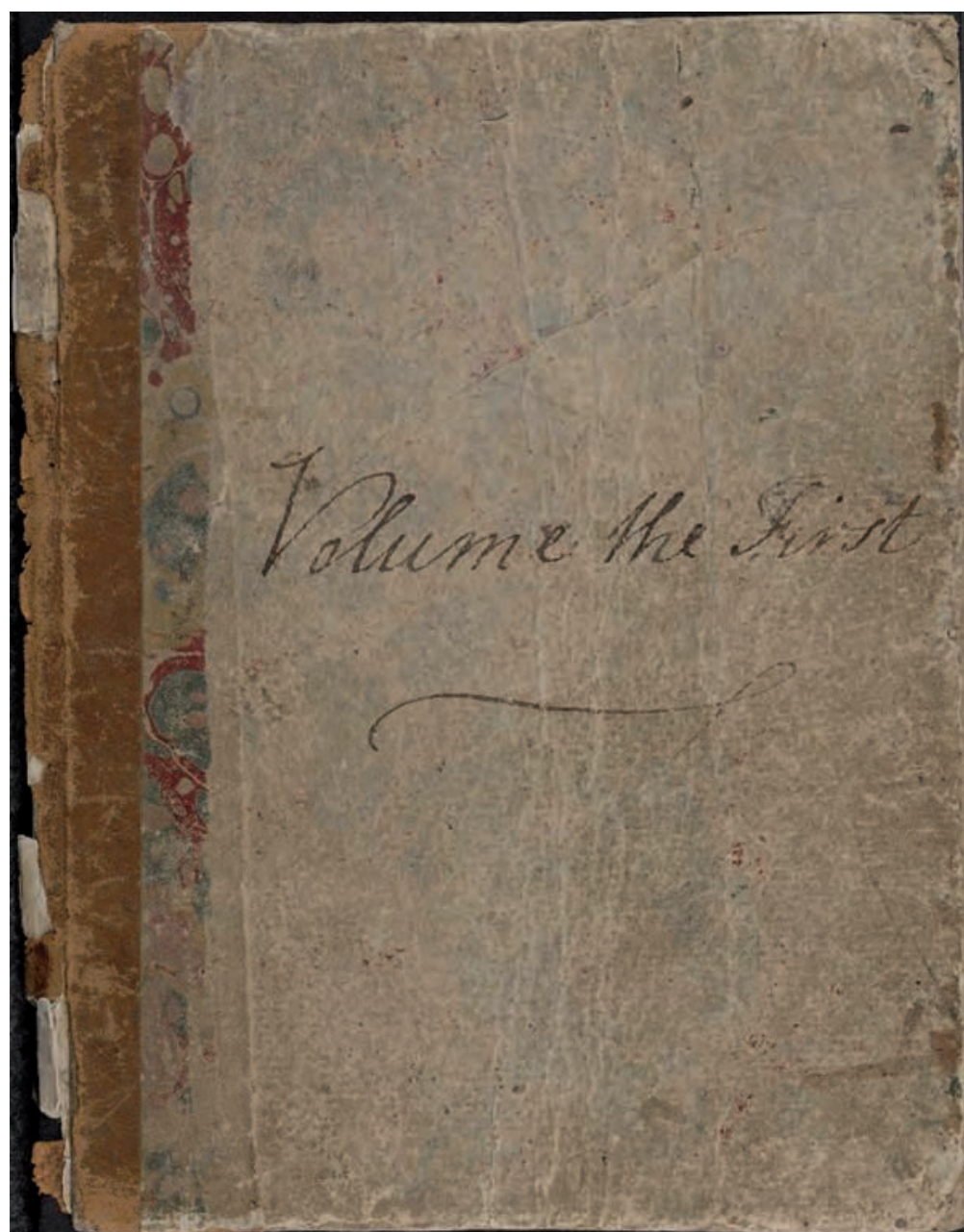

Fig. 1 : Jane Austen, Volume the First, couverture (Oxford Bodleian Library, Ms. Don.e.7) (C) Oxford Bodleian Library

3. L'auteur de l'article distingue d'une part les notebooks, qui sont des cahiers reliés et achetés dans le commerce, d'autre part les supports d'écriture confectionnés par Jane Austen elle-même : booklets (livrets) et gatherings, ces derniers étant des ensembles de feuillets pliés et placés l'un dans l'autre mais non reliés. Le français traduit usuellement gatherings par cahiers, ce qui crée dans la traduction une confusion avec les notebooks. C'est pourquoi nous utiliserons pour gatherings le terme de « cahier assemblé plié » (ou cahier plié), ce qui est du reste conforme au Vocabulaire codicologique de D. Muzerelle (note de la traductrice). 
Le fait d'écrire dans des cahiers reliés a encouragé la jeune Austen à présenter les mises au net de ses récits d'une manière livresque. L'aspect formel du cahier semble stimuler le façonnement bibliographique de ses textes verbaux, depuis l'imitation globale des effets de l'imprimerie qu'on retrouve dans les fictions publiées, jusqu'aux petits détails insignifiants, comme les tables des matières et la numérotation continue des pages de chaque cahier, qui est apparemment faite simultanément à la copie ; en revanche, elle ne pagine pas les brouillons qui nous sont parvenus. Il y a une relation évidente et intime, dans tous les manuscrits des cahiers, entre la présentation textuelle, la mise en page, le format et le statut du cahier. Ce sont des livres feints dans lesquels Austen joue et plaisante avec le format matériel et visuel autant qu'avec les conventions littéraires et le langage. Puisque leur aspect sur la page est à ce point important pour leur signification, l'accès aux documents ou aux supports autant qu'à leurs textes est souhaitable pour le lecteur moderne, que ce soit en fac-similés photographiques ou à travers une reproduction fidèle sous forme imprimée de leurs traits formels.

Ce ne sont pas les cahiers, où remaniements et corrections sont minimaux, qui révéleront les techniques de révision d'Austen, mais on peut y observer son aisance dans l'art de confectionner un livre, de simuler dans le manuscrit les régularités et les normes de l'espace standardisé de l'imprimé moderne. En effet, nous pourrions dire que dans les manuscrits en cahiers les conventions du livre ou de l'imprimé l'emportent sur la composition du texte. Par exemple, l'ensemble des morceaux contenus dans Volume the First, tous datables de 1787 à 1790 - Jane Austen avait entre 12 et 15 ans -, sont des paratextes (pages de titre, dédicaces et préfaces) plutôt que des textes. La dédicace «The beautifull Cassandra » (p. 115)4 (fig. 2) à Cassandra Austen, sa sœur, est une composition littéraire en soi, par rapport à laquelle l'histoire elle-même n'est rien de plus qu'une liste d'intitulés de chapitres (fig. 3). Pour une part, l'objectif en recopiant ces morceaux semble avoir été un amusement bibliographique : des préfaces plus longues que le texte qu'elles introduisent ; des pages de titre sophistiquées ; l'imitation de la convention de l'imprimé par l'emploi de guillemets tout au long de la marge de la page pour marquer le dialogue (par exemple, dans « Jack \& Alice », p. 33-35) - caractéristique typographique habituelle dans les romans de la sensibilité en Angleterre et en France à la fin du XVIII ${ }^{\mathrm{e}}$ siècle.
Parmi les artifices rhétoriques de prédilection utilisés dans Volume the First, les points de suspension sont également caractéristiques de l'imprimé au XVIII ${ }^{\mathrm{e}}$ siècle, employés par convention pour marquer l'incohérence ou la désintégration : une rupture de la communication. L'emploi par Austen d'une telle variété de codes venus de l'imprimé fait des écrits de jeunesse une sorte de jeu de société, à destination d'un public restreint et complice, où l'auteur et le lecteur (l'orateur et celui qui l'écoute) collaborent pour combler les vides et développer le texte.

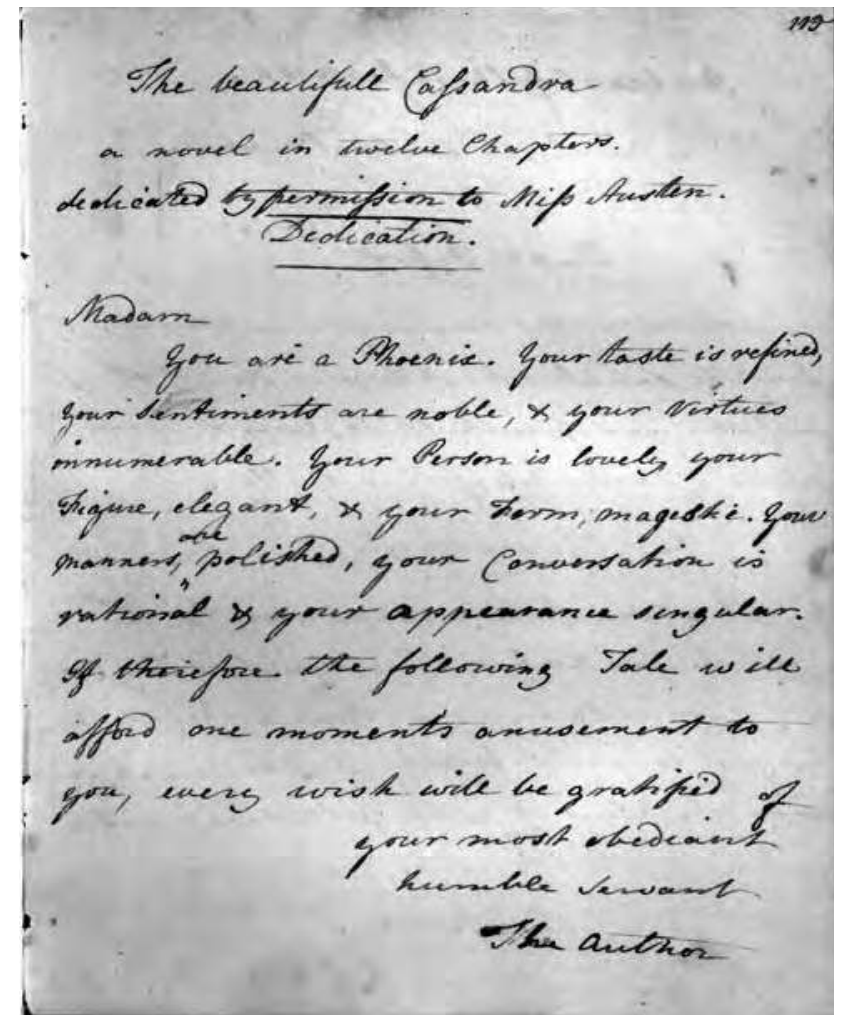

Fig. 2 : Jane Austen, Volume the First, p. 115 (Oxford Bodleian Library, Ms. Don.e.7) (C) Oxford Bodleian Library

4. Toutes les références dans le texte, par livret et par page, renvoient aux manuscrits de Jane Austen, tels qu'ils se présentent dans Jane Austen's Fiction Manuscripts: A Digital Edition, édités par Kathryn Sutherland (2010), disponible à l'adresse <www.janeausten.ac.uk>. Le lecteur intéressé pourra consulter les images numériques de chaque manuscrit sur ce site. 


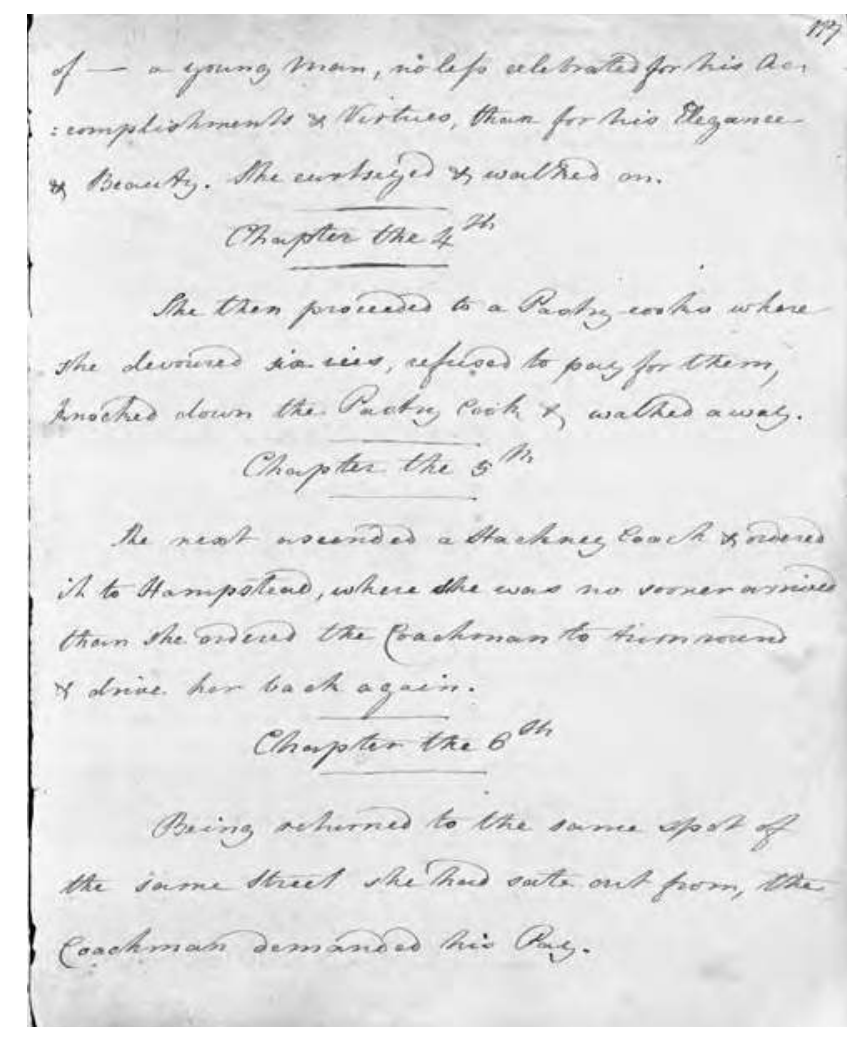

Fig. 3 : Jane Austen, Volume the First, p. 117 (Oxford Bodleian Library, Ms. Don.e.7) (C) Oxford Bodleian Library

Ces récits de jeunesse partagent sur le plan formel des traits communs avec certains recueils manuscrits des $\mathrm{XVII}^{\mathrm{e}}$ et $\mathrm{XVIII}^{\mathrm{e}}$ siècles, où une disposition imitant l'imprimé n'indique pas une intention d'être publié, mais plutôt une manière de qualifier l'espace manuscrit comme un espace de sociabilité, un espace partagé, et qui manifeste la détermination de l'écrivain dans la profession qu'elle s'est choisie $^{5}$. En dernier ressort, il s'agit de textes donnés à lire plutôt que de textes en voie d'écriture, de textes achevés, ou provisoirement achevés, avant d'être inscrits dans les cahiers ; ils sont en quête d'une reconnaissance sociale par la dédicace à la famille ou aux amis et plusieurs portent la trace d'une auctorialité collaborative ou d'une écriture à plusieurs mains 6 . Chacun des trois cahiers de jeunesse porte des marques d'usure prononcée, ce qui suggère de fréquentes relectures qui peuvent être datées avec certitude de la période antérieure à leur acquisition par les bibliothèques qui les conservent aujourd'hui, où leur accès a été limité.

\section{Les livrets faits maison (Homemade Booklets)}

Il est impossible de dater précisément le moment où Austen a commencé à préférer les cahiers assemblés pliés ou les livrets faits maison comme espace de rédaction de ses textes. Les brouillons des récits qui ont été ensuite rassemblés dans une copie au net sur cahier du commerce peuvent avoir d'abord pris forme dans les livrets faits maison. Le premier véritable manuscrit de fiction qui a survécu sous la forme d'un livret est The Watsons. Le papier est daté de 1803, et le manuscrit a probablement été ébauché en 18041805. The Watsons est écrit dans une série de onze livrets numérotés (fig. 4), qui après un début hésitant (un livret de quatre pages plié de manière non usuelle chez Austen pour adopter une orientation au format paysage large), se déploient dans des ensembles de huit pages, jusqu'au dernier livret qui compte quatre pages. Depuis The Watsons, c'està-dire lorsqu'Austen approche de la trentaine et jusqu'à la fin de sa vie, les brouillons qui nous restent possèdent certains traits communs. Le plus évident est leur espace d'écriture : ce sont de petits livrets faits maison, composés à partir de feuilles de papier pliées et coupées pour former des cahiers assemblés de quatre à quarante feuillets, mesurant approximativement $190 \times 120 \mathrm{~mm}$ (The Watsons et

5. À comparer par exemple avec les albums soigneusement recopiés des œuvres de jeunesse manuscrites de Lady Mary Pierrepont (la jeune Mary Wortley Montagu), à l'âge de 14 ans ou un peu plus, décrits dans l'article d'Isabel Grundy, « Lady Mary Wortley Montagu and her daughter: the changing use of manuscripts », Women's Writing and the Circulation of Ideas: Manuscript Publication in England, 1550-1800, dir. George L. Justice and Nathan Tinker, Cambridge, Cambridge University Press, 2002, p. 182-200 ; et I. Grundy, " "The Entire Works of Clarinda": Unpublished Juvenile Verse by Lady Mary Wortley Montagu », Yearbook of English Studies, n 7, 1977, p. 91-107, en particulier p. 97.

6. Il apparaît que cette collaboration s'est poursuivie lorsqu'Austen atteignit l'âge adulte, alors qu'elle était déjà un auteur publié. Le cahier de jeunesse Volume the Third contient des révisions et des expansions de ses récits qu'on peut dater de 1814-1816 environ des mains de la nièce et du neveu d'Austen. Voir Kathryn Sutherland, « From Kitty to Catharine: James Edward Austen's hand in Volume the Third », Review of English Studies, new series, n 66 (2014), p. 124-143. 


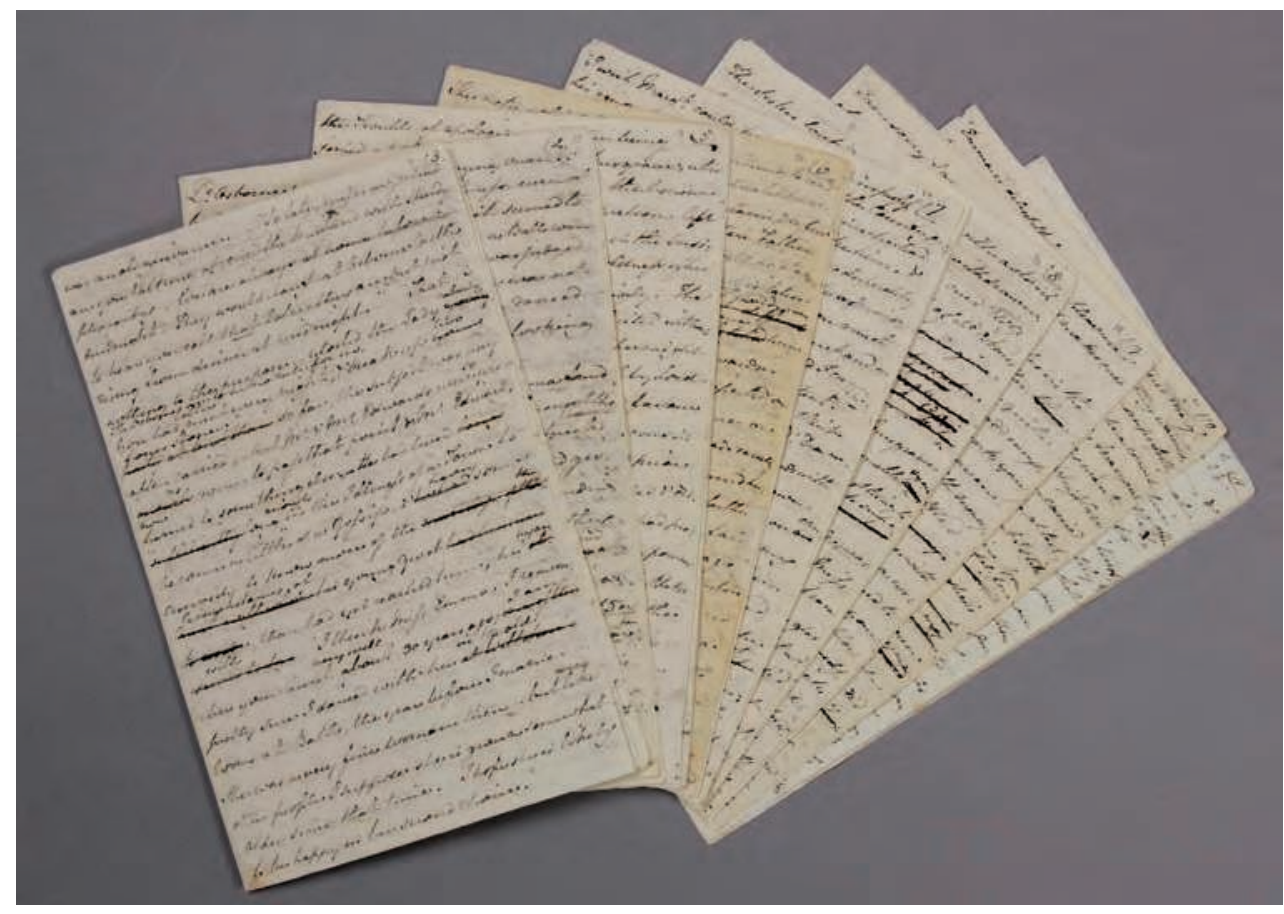

Fig. 4 : Jane Austen, The Watsons, onze livrets manuscrits (C) Oxford Bodleian Library

Sanditon), voire $155 \times 90 \mathrm{~mm}$ (Persuasion). Leur est aussi commune l'absence des marques qui apparaissent dans les textes somptueusement mis en page dans les cahiers du commerce. Dans les livrets, les brouillons n'ont aucune pagination, aucune marque paratextuelle, et font un usage parcimonieux des paragraphes. L'utilisation récurrente par Austen d'une gamme de mots contractés semble adaptée à ces espaces étroitement remplis. En somme, les textes comme les supports sur lesquels ils s'inscrivent, sont très comprimés. À la différence des livrets de The Watsons, ceux qui accueillent Persuasion et Sanditon distribuent leur texte en chapitres et datent certaines campagnes d'écriture, mais aucun de ces trois manuscrits ne porte de titre.

Ces livrets donnent des indices sur l'idée qu'Austen se faisait de son écriture. Pourquoi fabriquer des livrets ? Pourquoi ne pas composer sur des feuilles volantes ou directement sur des cahiers reliés du commerce ? Selon toute apparence, les livrets simulent la fabrication commerciale d'un livre imprimé, qui est composé d'une succession de cahiers, jusqu'au format de la feuille de papier, qui s'approche du format in-douze du roman publié. Chacun d'aspect modeste, ils ont une structure cumulative et répétitive qui imite l'accroissement du livre ; et cela dès le moment de la première rédaction. Étant de petit format, ils sont transportables et privés, relativement faciles à emporter avec soi en voyage et à soustraire à des regards curieux. Comme l'a rapporté l'histoire familiale d'Austen ou le mythe : «Elle écrivait sur de petites feuilles de papier qui pouvaient aisément être mises de côté, ou recouvertes d'un papier buvard7. » De manière encore plus significative, l'utilisation de livrets comme espace de rédaction implique une forte confiance en soi.

Dans un premier temps, Austen peut avoir choisi les cahiers du commerce comme un moyen de réunir, conserver et présenter à autrui ses écrits de jeunesse et avoir perçu seulement plus tard le potentiel du livret fait maison comme support de rédaction ; ou bien, comme les jeunes sœurs Brontë, elle peut avoir été attirée par la fabrication de livres en amateur dès son plus jeune âge, manifestation d'un talent précoce et aussi, dans son cas, d'un talent pour la parodie. Mais il y a une différence entre les deux usages en termes de conservation et de rédaction. C'est particulièrement le cas pour ce qui concerne l'écriture narrative. En tant que support de transcription et de mise au net, le cahier implique le dépôt de quelque chose de formé ; en tant que support pour l'évolution, la progression et les va-et-vient de la rédaction, le même espace suggère une forte certitude

7. James Edward Austen-Leigh, A Memoir of Jane Austen and Other Family Recollections, éd. Kathryn Sutherland, Oxford, Oxford University Press, 2002, p. 81. 


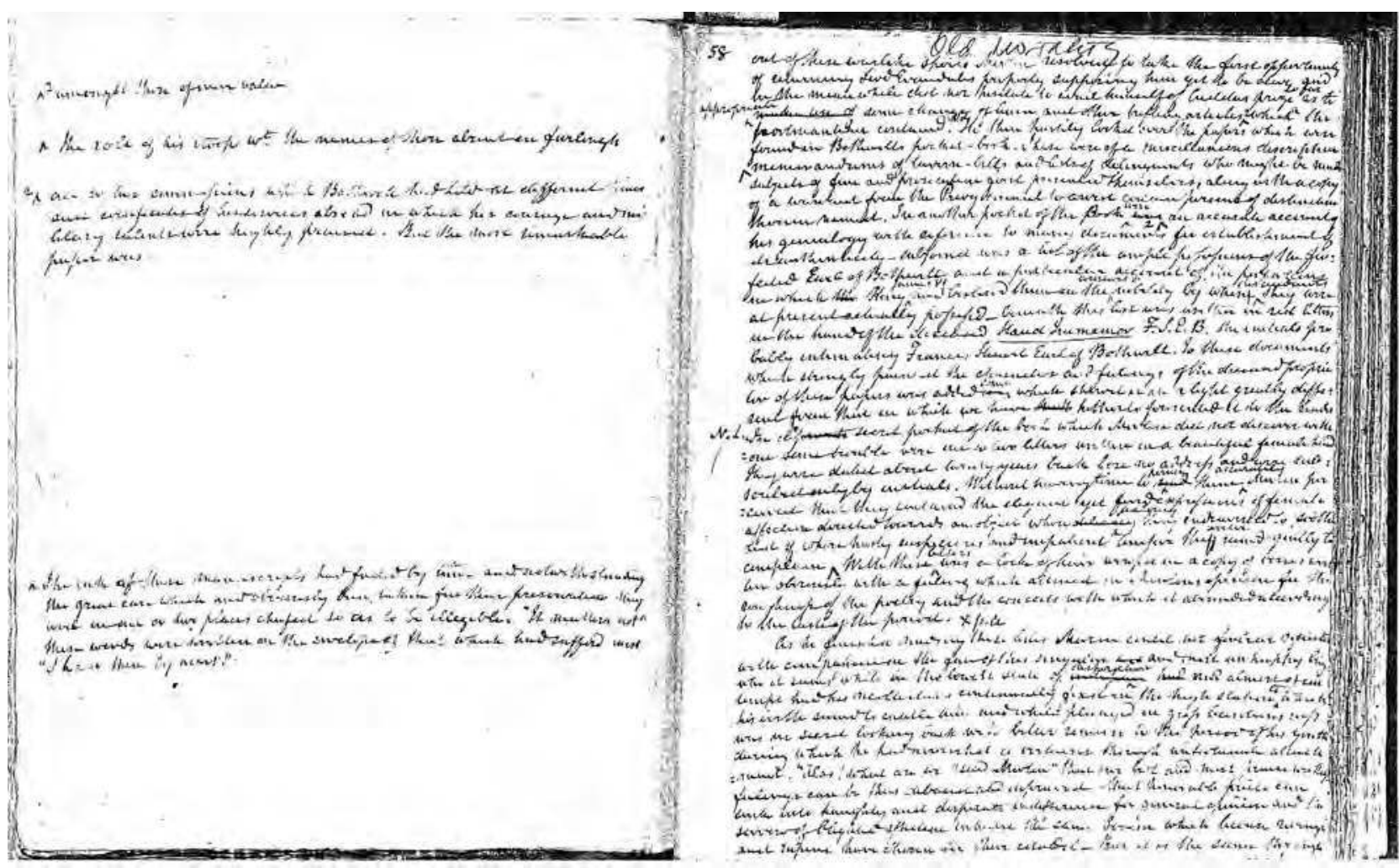

Fig. 5 : Manuscrit de Walter Scott, The Tale of Old Mortality c) New York, Morgan Library

de l'écrivain quant à la possibilité d'avancer son travail sous la forme d'une séquence dont les éléments sont liés les uns aux autres depuis le début dans le bon ordre (l'ordre définitif). Soit l'écrivain est sûr de lui, soit l'implacable séquentialité du support exerce sa propre tyrannie sur le récit, le poussant dans une seule direction et condamnant à un stade précoce d'autres options pour l'élaboration ou la génération plus libre du texte et selon un ordre dont le sens et la successivité doivent encore émerger. Bien sûr, l'écrivain peut supprimer du matériau, introduire de nouvelles lignes au sein de l'espace interlinéaire, pour réviser ou retravailler des choix antérieurs, mais la flexibilité est limitée. Les cahiers, à la différence des feuilles volantes, représentent et encouragent le flux en avant de l'écriture.

Une pratique usuelle chez les romanciers anglais du début du XIX ${ }^{\mathrm{e}}$ siècle consistait à utiliser les cahiers assem- blés pliés pour rédiger un brouillon entier, en employant le recto pour développer le corps du récit et en conservant les versos blancs en vis-à-vis pour les corrections ou la création de nouveaux matériaux. Ainsi utilisé, le verso introduit une sorte de fluidité, revisitant ce que le recto a trop tôt scellé. Son contemporain Walter Scott partageait le goût d'Austen pour ce type de cahier comme support de rédaction continue, imposant l'idée que sa structure séquentielle anticipe le récit non encore écrit et le pousse en avant ; mais lorsqu'il rédigeait, il suivait un schéma régulier, formant des livrets de quatre feuillets, remplissant chaque recto presque jusqu'au bord et utilisant le verso en vis-à-vis pour la révision et l'ajout (fig. 5). Le papier utilisé par Scott est aussi nettement plus grand (presque toujours $262 \times 205 \mathrm{~mm}$ ) que les minuscules feuilles employées par Austen. 


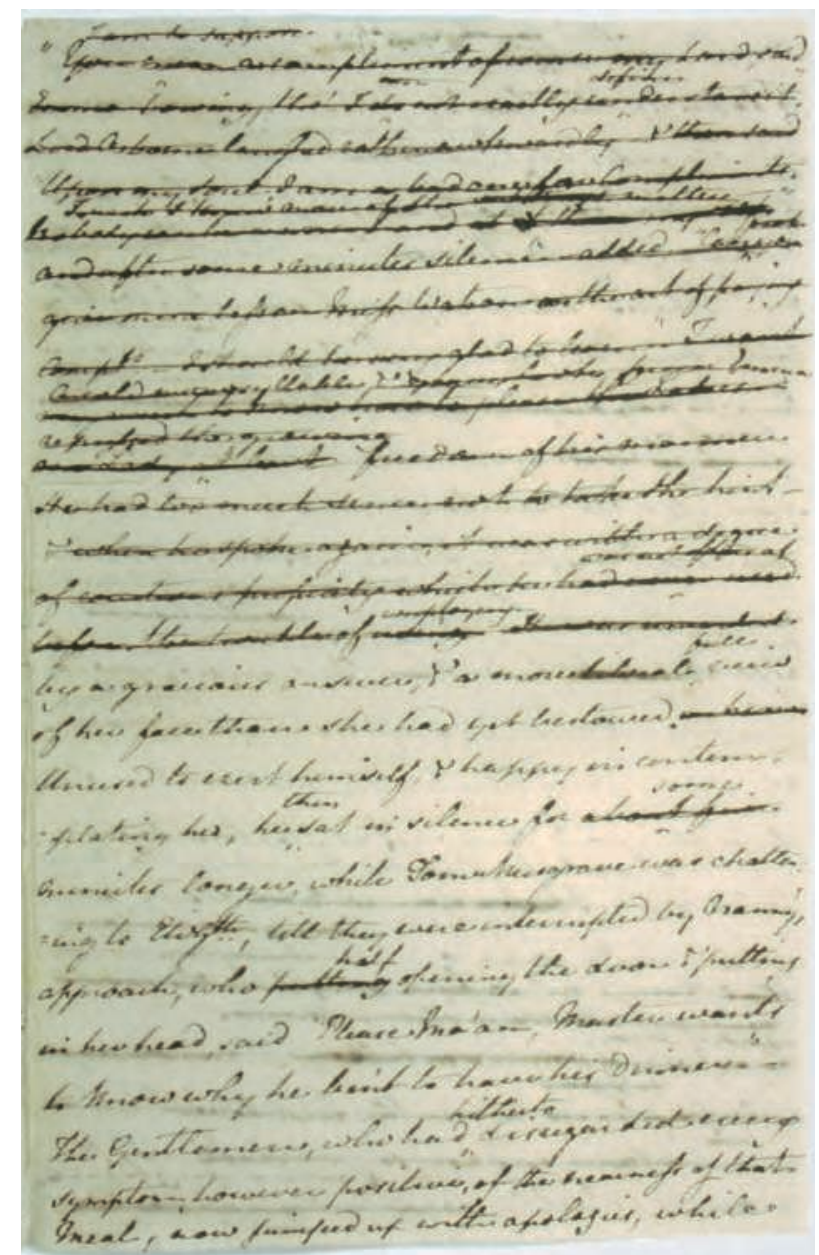

Fig. 6 : Jane Austen, The Watsons, livret 7, p. 7 (Oxford Bodleian Library, Ms. Eng.e.3764) (C) Oxford Bodleian Library

En comparaison, la méthode d'Austen semble être bien plus risquée et audacieuse. Ce qui frappe immédiatement est la densité avec laquelle elle remplit son support, restreignant apparemment tôt ses options. Tous ses brouillons de travail sont couverts d'une écriture très serrée ; les lignes sont compactes ; chaque page est remplie, recto et verso, jusqu'au bord, ne laissant aucune place pour des remaniements de grande ampleur, laissant l'impression qu'une fois qu'elle a jeté sur le papier ce qu'elle avait à écrire, elle n'envisage pas de le retravailler de fond en comble, ni de réordonner le fil de l'histoire, les descriptions ou les dialogues. Les révisions se font dans l'interligne, écrasées dans un espace qui ne leur était pas destiné (fig. 6). N'ayant pas prévu d'espace libre pour accueillir des repentirs de quelque ampleur, Austen a dû, dans les rares cas où elle a décidé de raturer et de réécrire substantiellement pour réorienter ou rouvrir le récit, coller littéralement des morceaux de papier sur le brouillon. Dans The Watsons, on trouve trois morceaux épinglés sur la page, découpés avec une certaine précision pour s'ajuster aux endroits qu'ils doivent remplir, qu'il s'agisse de remaniements ou d'extensions. Dans Persuasion, un seul morceau de papier (à la page 19a) (fig. 7), découpé dans la partie laissée vide du volet destiné à recevoir l'adresse d'une lettre, est fixé avec des cachets sur un passage lourdement raturé (à la page 19, fig. 8), et ailleurs dans ce petit cahier de deux chapitres, un ajout tardif est marqué par un grand $\mathrm{X}$ à l'endroit où il doit être inséré (plusieurs pages avant).

Les révisions parfois intenses et laborieuses et la pratique du papier collé conduisent à révoquer l'idée que chaque manuscrit d'Austen est passé par l'établissement de plusieurs versions manuscrites : ces manuscrits conjuguent semble-t-il en un seul objet compact des versions primitives et tardives. Deux des manuscrits d'Austen qui sont parvenus jusqu'à nous - The Watsons et Sanditon - sont peut-être des brouillons uniques, retravaillés en différentes occasions. Nous ne pouvons pas savoir si, dans le cas où ils auraient été achevés, Austen en aurait fait une copie intégrale. Des signes d'hésitation dans leurs sections d'ouverture, suivis par des développements plus lisses (la conversation semble venir plus facilement à Austen), impliquent à la fois des révisions immédiates, contemporaines du moment de la création, et des changements survenus plus tard. Les chapitres éliminés de Persuasion ont peut-être d'abord été une mise au net, mais on voit qu'ils subissent rapidement la pression des repentirs et d'un développement plus complexe.

Selon toute évidence, Austen employait le papier avec parcimonie ; et cette économie peut avoir eu des motivations autres que financières. La célèbre description de sa manière de travailler - « le petit morceau (large de deux pouces) d'ivoire sur lequel je travaille avec un pinceau si fin que même beaucoup d'effort fait peu d'effet »-se trouve dans une lettre à son neveu James Edward Austen de décembre 1816. Elle s'y dénigre, en contraste avec ce 


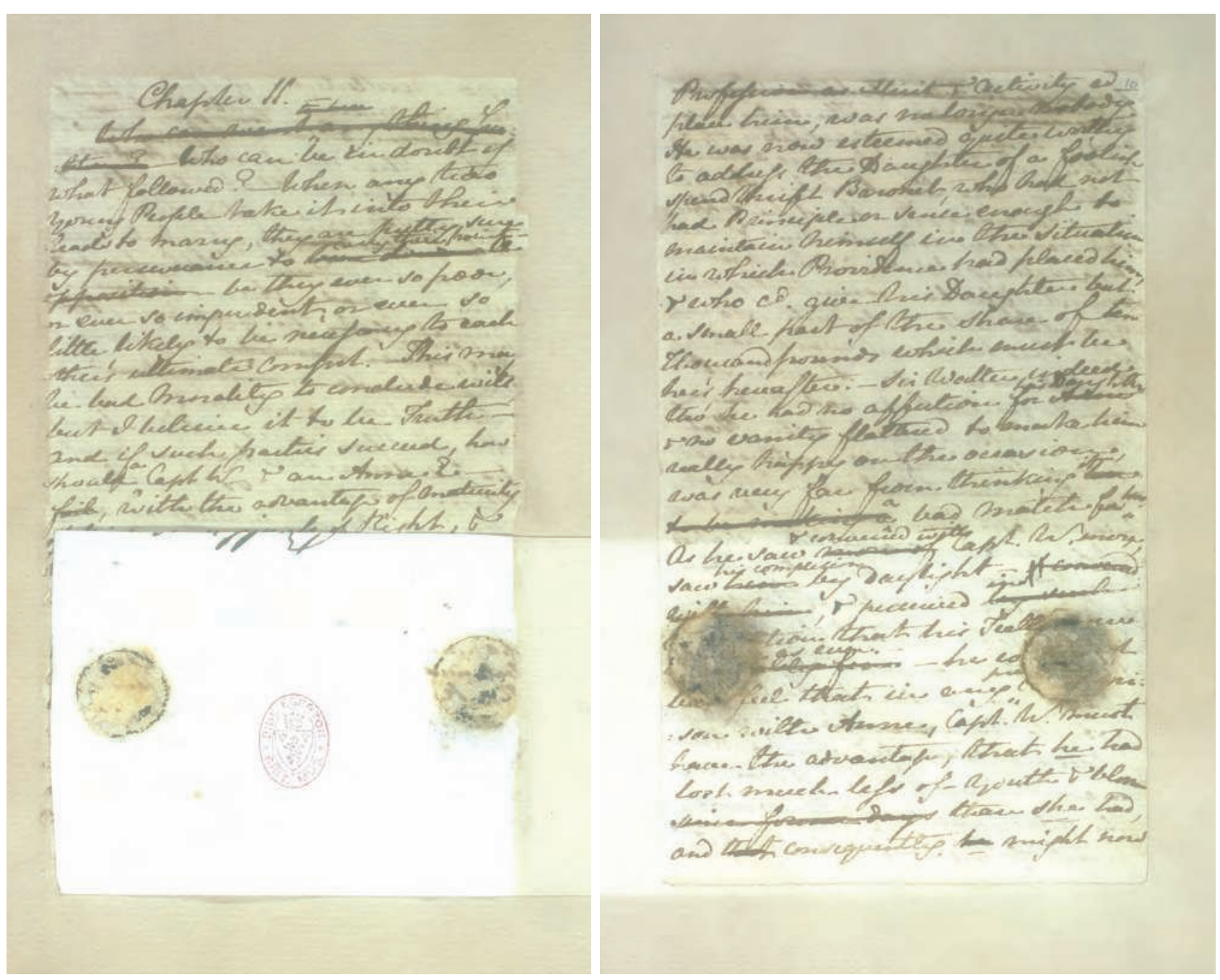

Fig. 7 et 8 : Jane Austen, Persuasion, p. 19a et 19 (London British Library, Ms. Egerton 3038) (C) London British Library

qu'elle dit de lui à l'époque, de ses « esquisses fortes, viriles, spirituelles, pleines de variété et d'éclat »-il essayait lui-même d'écrire un roman. En général ce commentaire est cité par les critiques pour expliquer les sujets intimistes d'Austen (ce qu'elle décrit ailleurs de la façon suivante : «3 ou 4 familles dans le village d'un comté - le vrai sujet sur lequel travailler », septembre 1814 à Anna Austen, pendant la rédaction d'Emma ${ }^{8}$. Mais cette description vaut encore mieux pour évoquer son support d'écriture couleur ivoire, son papier, et sa méthode d'écriture. L'intense concentration offerte par les petits livrets et le défi de fixer sa vision à travers l'objectif le plus étroit possible peuvent avoir donné un aiguillon vital à sa créativité : l'espace restreint de la petite page remplie à ras bord, l'unique brouillon maintes fois retravaillé, peuvent avoir été les conditions indispensables à son art dépouillé. En d'autres termes, les petits cahiers pliés peuvent avoir été les équivalents matériels d'une économie de l'expression créatrice. Les écrivains sont souvent des individus aux habitudes obsessionnelles. La structure matérielle récurrente et le mode de construction des supports d'écriture d'Austen suggèrent que cette habitude était dans son cas une discipline essentielle pour sa pratique rédactionnelle.

Les petits cahiers aux feuilles de papier pliées étaient confectionnés à la maison à l'imitation des cahiers d'imprimerie. Dans leur matérialité, ils livrent des indices sur la manière de travailler d'Austen et montrent comment sa relation avec les matériaux d'écriture a évolué dans le temps.

8. Jane Austen's Letters, éd. Deirdre Le Faye, 3 e édition, Oxford, Oxford University Press, 1995 , p. 323 et 275. 
The Watsons, un manuscrit d'environ dix-sept mille cinq cents mots, est divisé en onze minces sections. Chacune est constituée d'une demi-feuille d'un papier à écrire postquarto (environ $244 \times 193 \mathrm{~mm}$ ), plié puis coupé encore une fois en deux, une moitié insérée ensuite dans l'autre afin de former un livret de quatre feuillets ou huit pages. D'après la position régulière des filigranes dans chaque livret, il est clair qu'elle avait seulement des demi-feuilles, achetées chez le papetier. Il est également clair, d'après l'emplacement récurrent des filigranes dans chaque livret, qu'il n'y a pas eu de papier jeté ou gâché : chaque livret est construit exactement de la même manière. En d'autres mots, aucun feuillet ni bifeuillet n'a été perdu. Enfin, il apparaît également que les trois papiers collés (dans les livrets 7, 9 et 10) ont été découpés à partir d'une seule et même feuille de papier. D'après leur orientation variable par rapport à la feuille d'origine, ces papiers collés ne peuvent avoir été annotés qu'après le découpage ; et pourtant les textes qu'ils contiennent ont été calibrés à l'avance et ils sont remplis avec une extrême précision. Cette épargne dans l'usage du matériau témoigne d'une économie et d'une sûreté remarquables dans la rédaction.

Même si on retrouve les mêmes phénomènes dans les manuscrits de Persuasion et de Sanditon, chacun de ces livrets plus tardifs est considérablement plus épais. On a l'impression qu'entre The Watsons, vers 1804, et Sanditon, en 1817 , les cahiers pliés qui servent de brouillon prennent de l'ampleur, ce qui suggère une confiance croissante de l'auteur quant à sa capacité de travailler sur une unité narrative plus longue, au sein d'un support matériel plus vaste mais avec des possibilités de révision limitées. On constate également qu'à la différence des livrets de The Watsons, ces derniers livrets étaient cousus, ce qui rendait plus difficile de retirer des pages. Ce n'est pas tout, les plus petites unités qu'on trouve dans The Watsons sont le plus souvent structurelles : bien que chaque livret ne contienne pas une section narrative complète - le manuscrit est à la fois dépourvu de paragraphe et sans division en chapitre -, Austen utilise néanmoins régulièrement les limites des petits cahiers pliés pour faire aboutir les éléments qui composent l'unité de sens qui s'y trouve. Par contre, le livret de Persuasion emploie ses trente-deux pages pour donner la mesure à et pour structurer deux chapitres entiers. Bien que les cahiers pliés du manuscrit de Sanditon soient plus longs, Austen semble avoir un besoin moins pressant de parvenir à ce type de clôture locale.

Le plus épais de tous est le dernier cahier plié d'Austen : le livret 3 de Sanditon, avec quatre-vingts pages, soit huit de moins que le manuscrit entier de The Watsons. Il est aussi le plus vide. Il se termine par une ligne écrite en haut de la page $40\left(f^{\circ} 20 \mathrm{v}^{\circ}\right)$, suivie de la date du 18 mars, exactement au milieu du cahier. Les quarante dernières pages sont vierges. Pourquoi son dernier cahier était-il le plus épais de tous ? Les preuves manquent pour affirmer que les livrets de rédaction d'Austen se sont épaissis entre 1804 et 1817 . Les manuscrits qui demeurent donnent cette impression, bien sûr, mais trop de choses manquent. Simplement il se peut que, bien que le brouillon de The Watsons soit complexe, à strates multiples, il ne constitue pas une œuvre ayant atteint un stade tel qu'elle pouvait être envoyée à l'imprimeur, comme c'est le cas pour les chapitres supprimés de Persuasion. Les épais livrets de Sanditon peuvent refléter la confiance d'un auteur désormais aguerri et publié ; ou bien le dernier cahier, le plus épais de tous, peut traduire une sorte de défi lancé à la maladie qui allait bientôt emporter Austen. Mais l'explication peut être d'ordre pratique. À ce stade l'auteur, proche de la fin, avait des difficultés à rester longtemps assise à sa table ; un cahier de quatre-vingts pages pouvait à lui seul constituer une sorte de petite table portative.

À la différence des brouillons des œuvres de ses contemporains anglais - des poèmes de Keats ou de Wordsworth, ou du roman de Mary Shelley, Frankenstein - les manuscrits d'Austen ne sont pas des formes de prépublication ou des formes avant-textuelles 9 d'œuvres qui trouvent leur véritable achèvement en tant que textes publics et à travers l'impression. Les cahiers de jeunesse ne sont en rien des brouillons, mais des publications limitées au cercle privé ou confidentiel ; quant à The Watsons et à Sanditon, leur inachèvement est problématique ; et à l'exception de quelques pages, les chapitres de Per-

9. Concernant l'« avant-texte », terme courant en théorie génétique et dans les études textuelles (qu'on associe aux états de composition du texte, avant publication), voir Louis Hay, « Does “Text” Exist? », Studies in Bibliography, $\mathrm{n}^{\circ} 41,1988$, p. 64-76. 
suasion sont l' «avant-texte» achevé d'un « texte » qui n'a pas été imprimé. Et puisque, sans dossier manuscrit conservé, les six romans achevés n'existent que sous leur forme publique pour laquelle aucun avant-texte n'est disponible, c'est seulement indirectement que les œuvres manuscrites peuvent éclairer l'étude des romans publiés de Jane Austen.

Cependant, on observe au long des pages manuscrites des manies et des rythmes rédactionnels, qui vont de tics stylistiques presque involontaires à des mouvements plus amples, phases d'effort et de jaillissement, éclairant le type d'écrivain qu'était Jane Austen. Il n'est pas étonnant, si l'on garde à l'esprit l'extrême discipline imposée par le support d'écriture, de découvrir qu'Austen écrit avec économie, la rédaction émergeant par la répétition. «Amiable », «agreable », « disagreable » sont ses adjectifs préférés, largement utilisés dans tous les manuscrits ; « extraordinary » est abondamment repris dans Sanditon (livret 2, p. 10, 15, 24, 33 ; livret 3, p. 10, 23, 24), roman où Austen se livre également à de nombreuses expérimentations verbales. Elle engrange et réemploie les expressions et les sujets, de même que les péripéties et les intrigues ; au point que des groupes de mots reviennent régulièrement à l'oreille, tels des semi-échos. Le « old Coachman » qui « will look as black as his Horses » dans The Watsons (livret 6, p. 3), est évoqué douze ans plus tard dans la page d'ouverture de Sanditon, où le cocher est décrit comme «looking so black, \& pitying \& cutting his Horses » (ensuite raturé). La répétition est pratiquée à large échelle. Au niveau le plus élémentaire, un simple mot est biffé, un autre testé à sa place, jusqu'à ce que le premier soit rétabli. Par exemple :

\footnotetext{
«Postboy» $\rightarrow$ « Driver $\rightarrow$ «Post-Boy» (The Watsons, livret 8, p. 5);

«unfit $\rightarrow$ unsuitably $\rightarrow$ unfit $»($ livret 10, p. 1$)$;

«party» $\rightarrow$ « circle $\rightarrow$ « party $»($ livret 10, p. 2$)$;

«thought $\rightarrow$ «found $\rightarrow$ «thought $»$ (Sanditon, livret 3, p. 17).
}

Ailleurs, de l'échelle du mot on passe à celle de la phrase, et nous voyons comment, à partir d'une séquence de mots, posés là, disloqués, puis replacés ailleurs se développent une forme et un motif plus vastes. Prenons pour exemple la première phrase du manuscrit de Persuasion, p. 24 :
1) He quitted Bath soon after and on Mrs Clay's following <him $>\rightarrow \mathrm{He}<$ soon> quitted Bath; and on Mrs Clay's $<$ quitting it likewise $>$ soon after $<$ wards $>$.

2) it was pretty evident on what terms they had previously been $\rightarrow$ it was evident $<$ that he had been playing a double game $>\rightarrow$ it was <evident how double a Game he had been playing $>$.

On le voit, la rédaction émerge comme un processus de réitération imposant, peu à peu, un rythme qui structure, équilibre et étend la phrase.

Il faut aller plus loin pour rendre compte du taux relativement faible de révision dans les brouillons, lorsqu'il s'agit de transcrire la conversation et le discours intérieur ou la réflexion, en comparaison avec l'effort plus grand fourni pour établir les relations spatiales : la manière dont les personnages se tiennent les uns par rapport aux autres; la manière dont ils se déplacent ; ce qu'ils voient ou entendent là où ils sont assis ou debout; quels objets les entourent. La différence laisse supposer que pour capter le langage parlé, la manière d'écrire d'Austen était plus proche de l'enregistrement ou de la transcription du son. En particulier, les passages qui semblent couler de sa plume supposent un acte compositionnel précédant l'écriture, suggérant qu'Austen suivait la dictée d'une oreille intérieure, que pour elle la composition était « phonocentrique 10 ». Cela n'est pas surprenant : en dépit de l'emprise de l'imprimé, le roman tel qu'elle l'a développé est conversationnel et la conversation, à la différence de la prose, ne dépend pas de l'écriture ${ }^{11}$. Quelque chose qui relève du pouvoir de cette oreille intérieure, et sa probable association avec la création mentale d'un personnage par le langage parlé, est saisi dans le témoignage de deux nièces, Marianne et Louise Knight, qui, évoquant probablement la visite d'Austen à Godmersham à l'automne 1813, racontèrent bien plus tard le souvenir d'avoir vu leur tante Jane écrire :

10. À rapprocher des arguments qui proposent de voir en Wordsworth un écrivain « phonocentrique » chez Mary Jacobus, à partir de Derrida, De la grammatologie, dans Romanticism, Writing, and Sexual Difference, Oxford, Clarendon Press, 1989, p. 169 ; et chez Sally Bushell, Text as Process: Creative Composition in Wordsworth, Tennyson, and Dickinson, Charlottesville/London, University of Virginia Press, 2009, p. 100-101. 11. Voir Ann Banfield, Unspeakable Sentences: Narration and Representation in the Language of Fiction, Boston/London, Routledge Kegan Paul, 1982, qui (après Otto Jespersen) décrit le langage de la fiction narrative comme « du discours et de la pensée représentés ». 
Tante Jane se tenait assise, silencieusement à l'ouvrage [cousant] à côté du foyer dans la bibliothèque, se taisant pendant un bon moment, et puis soudain éclatait de rire, se dressait en sautant et traversait la pièce en courant jusqu'à une table où il y avait des plumes et du papier, écrivait quelque chose et retournait près du feu, reprenant tranquillement son ouvrage comme auparavant 12 .

Les pages des chapitres de Persuasion manifestent une énergie nerveuse caractéristique d'une composition phonocentrique : elles donnent l'impression de respirer ou de s'ouvrir en même temps que la voix qui parle ou qui réfléchit, procure une illusion de spontanéité (d'une composition préécrite), avant de se contracter, de se couvrir de réécritures, avec de minuscules ou plus rarement d'amples ajustements dans les passages intermédiaires de description et d'explication narrative. Ce type de rédaction est caractérisé par un emploi massif du tiret (fig. 9). Dans The Watsons, qui est le brouillon le plus densément retravaillé, les mots parlés sont souvent tout ce qui survit sans altération d'une campagne de réécriture, tandis que la langue est davantage susceptible d'être démantelée et réécrite si elle s'éloigne de l'idiome conversationnel et si elle se rapproche de l'apport d'informations. Par exemple le livret 4, p. 5, garde intactes les remarques légères des danseurs dans les salles de réunion, tandis qu'autour d'eux la scène est réarrangée de manière troublante, ses espaces rendus plus spécifiques et décrits avec une attention au détail inhabituelle, voire déformante, ce qui a pour effet de construire à travers les objets une image de contrainte mentale, avec l'héroïne Emma Watson en son centre.

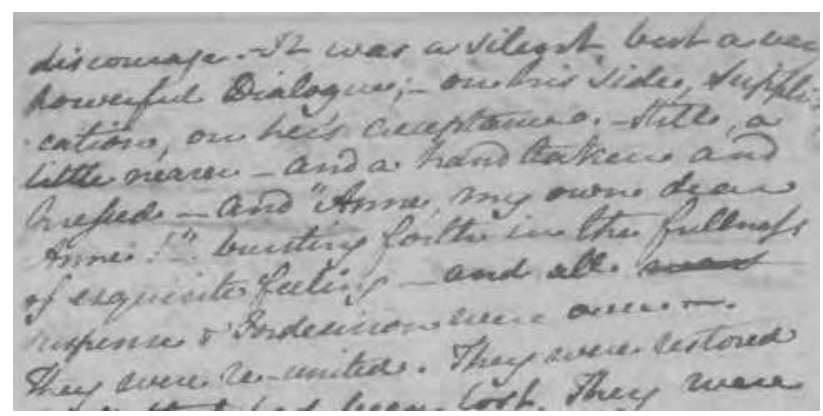

Fig. 9 : Jane Austen, Persuasion, p. 11

(London British Library, Ms. Egerton 3038) (C) London British Library
Dans Sanditon, la communication conversationnelle et l'organisation narrative se brisent chacune sous l'effet des obsessions d'une gamme de personnages excentriques. Leur discours, qui prend le plus souvent la forme de monologues, surgit régulièrement déjà précomposé sur la page. Les excentriques du roman sont pleinement dotés d'une voix assurée dont les divagations sont presque complètement textualisées quand elles surgissent sur le papier. La lettre (chapitre V, livret 2, p. 12-15), qui fait entrer en jeu pour la première fois le personnage hypochondriaque de Diana Parker, est une extraordinaire mise en scène de soi. La force de ce portrait tient dans l'assurance avec laquelle la voix détermine la personnalité, ce qui se reflète dans une impeccable présentation graphique sur la page manuscrite et dans l'aisance avec laquelle est obtenu un effet d'étrangeté : sur quatre-vingt-neuf lignes, il n'y a que treize corrections mineures, dont la plupart rapprochent la voix encore plus près de l'expression orale.

Dans l'examen des manuscrits de travail d'Austen, la question du déroulement temporel des opérations d'écriture est cruciale, parce qu'il est intimement lié à la conceptualisation et à la composition, à une manière de travailler envisagée à son niveau fondamental. Dans les brouillons, il y a toujours une plus grande fluidité, qui fait de la page non seulement un espace pour l'écriture mais aussi, à la différence de la page imprimée, un lieu de succession temporelle où nous pouvons voir les étapes de la rédaction à travers lesquelles l'écriture se construit en s'appuyant sur ses propres avancées. Le système général

12. Les souvenirs de Marianne Knight concernant sa tante Jane Austen ont été publiés pour la première fois d'après le récit d'une cousine à laquelle elle les raconta, dans Constance Hill, Jane Austen. Her Homes and Her Friends (1902, nouvelle édition, London/New York, John Lane, 1904, p. 202). Chacun est inclus dans Deirdre Le Faye, Jane Austen: A Family Record, Cambridge, Cambridge University Press, 2004, p. 206. 
qu'on observe dans les brouillons d'Austen - des passages plutôt nets, sans corrections, interrompus par des sections densément remaniées - suggère non seulement que certains actes de genèse (la transcription des conversations) prenaient forme (dans sa tête) avant le moment de l'écriture, mais aussi que, en tant qu'écrivain, Austen était un auteur à processus (ou immanent) plutôt qu'à programme. C'est-à-dire qu'elle écrivait spontanément, en comptant peu sur des plans conçus à l'avance ou sur des notes (aucun n'a été conservé); et même si l'écriture évolue de manière fragmentaire, ces fragments sont déjà pleinement textualisés dès le départ, prenant de l'ampleur depuis le lieu qu'ils occupent à travers des strates de réécriture ${ }^{13}$. Cette manière de réécrire, depuis l'intérieur du processus de rédaction, diffère de celle où la main qui révise revient pour mettre à jour un détail, améliorer une expression, ou ajuster le déroulement d'une histoire une fois celle-ci portée à son terme. Bien sûr, les romans, à la différence de brefs poèmes lyriques, ont peu de chance de prendre forme à partir de l'accumulation de morceaux nés d'une écriture spontanée, sans une anticipation de leur structure ou une conceptualisation plus large. Il doit $\mathrm{y}$ avoir programmation, à petite ou grande échelle. Ce que l'on observe dans les manuscrits d'Austen subsistants révèle plutôt une tendance qu'une explication complète de sa manière de travailler.

Certains passages et leur révision suggèrent qu'il y a eu planification et une vue d'ensemble sur le déroulement général, une méthode bien articulée, où une intention précise est poursuivie dans le temps, mais à quelle échelle ? Est-ce que les modifications d'un passage sont le fruit des changements d'idées et d'intentions à différents moments d'une unique séance, ou bien des altérations faites au moment d'une relecture ultérieure ; et combien de temps après ? le même soir? le jour suivant? la semaine d'après ?

Malgré des indices de planification, la disposition sur le papier des première et seconde (éventuellement troisième) tentatives pour formuler un mot, une phrase ou un développement plus longs, et la tendance, là où la révision est la plus chargée en repentirs, à aller directement vers une nouvelle version sans correction, sont la preuve que la plupart des remaniements dans Sanditon étaient spontanés, dans la continuité ou à peine dissociés du premier jet, sans que rien n'indique que des portions du texte aient été mises de côté et travaillées ultérieurement. On pourrait s'attendre à ce que l'introduction d'un nouveau personnage connaisse une première rédaction qui lui donne une structure préalable. Mais il est clair, d'après un passage où Mr Parker esquisse les traits du caractère de son frère Sidney (chapitre IV, livret 2, p. 5) (fig. 10), qu'il s'agit d'un passage complexe d'écriture à processus où la création, la correction et une création nouvelle jaillissent graphiquement en un seul acte d'écriture, ainsi qu'on peut le voir par l'effacement de certaines lignes remplacées par des matériaux à l'interligne supérieur et l'émergence d'un nouveau texte, tandis que des repentirs ramènent à la version de base.

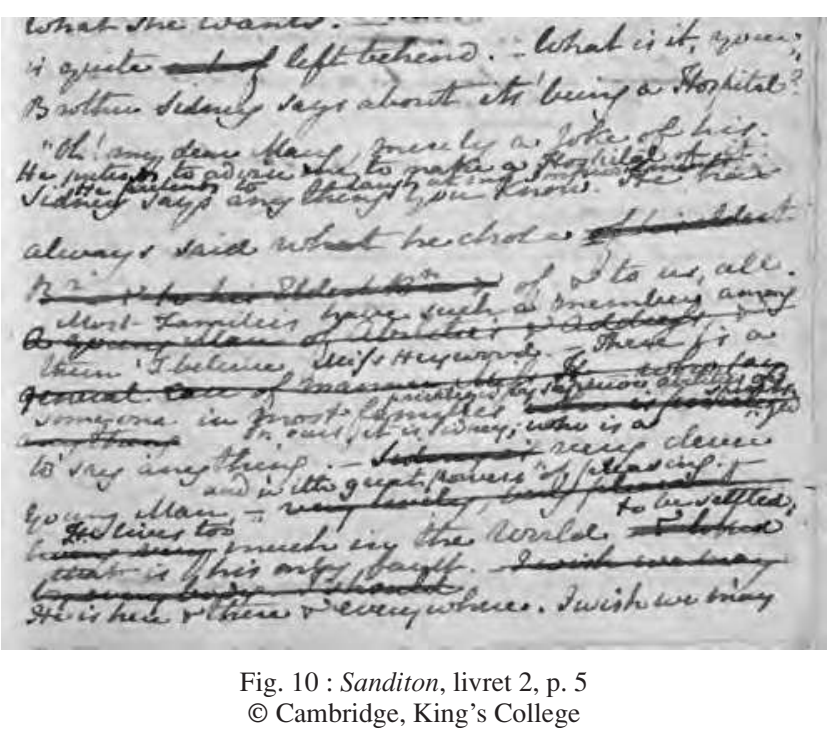

The Watsons aussi comporte beaucoup d'exemples de cet effort intense pour se construire ainsi de l'intérieur. Un exemple, tiré du livret 2, p. 4, montre Austen découvrant et ajustant selon ses propres circonvolutions mentales le

13. Concernant Austen en tant qu'écrivain immanent, je me fonde sur la distinction entre « immanent et «programmatique » proposée par Louis Hay, « Die dritte Dimension der Literatur: Notizen zu einer "critique génétique" ", Poetica, n 16, 1984, p. 311-314. Voir aussi Kathryn Sutherland, Jane Austen's Textual Lives: From Aeschylus to Bollywood, Oxford, Oxford University Press, 2005, p. 127-128; au sujet d'une distinction générale entre les formes d'écriture « à programme » et «à processus », voir S. Bushell, op. cit., p. 81-82. 
mélange d'émotions subtilement gradué qui constitue la réaction d'Emma Watson à sa nouvelle vie au sein d'une famille qu'elle connaît à peine. La phrase qui commence par «Her conversation with Eliz. had given her many unpleasing feelings to add to the awkwardness of so slight an acquaintance » est mise en pièces et réassemblée, avec un détour décisif au mot «impressions » où, ainsi que le révèle la fusion du texte principal avec ce qui est à l'interligne supérieur, la réécriture amène à la première rédaction. Le processus implique, en un seul acte créatif, la suppression, l'essai de différentes expressions et le déploiement des nuances de la situation émotionnelle avant le retour tardif aux termes initiaux :

Her conversation with Eliz. had $<$ too $>$ given/ing her many $<$ some very $>$ unpleasing/ant feeltings, to add to the awkwardness of so < with respect to her own family; \&/had $>$ slight an aequaintanee to the eonseiousness $<$ made her more open to any other disagreable im $>$ of $:<\&$ in particular to $>$ pressions, $<$ from any other cause, $>$ the awkwardness $<\&$ encreased her sense of the awkwardness $>$ of < rushing into Intimacy on $>$ so slight an acquaintance. seemed a serious Evit (The Watsons, livret 2, p. 4, lignes 3-8).

Le développement de la phrase peut être rendu de la manière suivante :

1) version initiale : «Her conversation with Elizabeth had given her many unpleasing feelings, to add to the awkwardness of so slight an acquaintance »;

2) version suivante : "Her conversation with Elizabeth had given her some very unpleasant feelings, with respect to her own family; \& the awkwardness of rushing into Intimacy on so slight an acquaintance seemed a serious Evil »;

3) version finale : "Her conversation with Elizabeth too giving her some very unpleasant feelings, with respect to her own family; had made her more open to disagreable impressions, from any other cause, \& encreased her sense of the awkwardness of rushing into intimacy on so slight an acquaintance. »

L'emploi et le réemploi de «awkwardness », écrit et effacé deux fois avant de trouver sa bonne place et avant que la phrase trouve sa conclusion, donne la mesure de la longueur et de la complexité du travail de construction intérieure qu'Austen a dû mener. Nous voyons comment, en construisant sa phrase, Austen établit une structure, la modifie puis rétablit les termes du départ à l'intérieur de son extension.

Pour ce qui concerne les remaniements considérables qu'on trouve notamment dans Persuasion et dans The Watsons, Austen a soigneusement découpé des morceaux de papier sur mesure. Elle les épingle ou les colle à la bonne place pour cacher ou remplacer un passage lourdement retravaillé ou raturé, ou bien pour insérer un développement ou un ajout substantiel. Alternativement, elle utilise une fois dans Persuasion une portion du document placée plus loin, marquant le nouveau texte par un grand $\mathrm{X}$ correspondant à l'endroit où il devait venir s'insérer, treize pages plus tôt. Quelle que soit sa méthode, dans tous les cas conservés, et en raison de la parcimonie dont elle fait preuve en remplissant sa page, l'expansion ou la révision du texte est caractérisée par un refaçonnement extrêmement poussé de la structure physique du brouillon, ce qui suggère combien il était inhabituel pour elle de prévoir et de laisser un espace pour la révision.

La fin écartée de Persuasion offre un cas d'école sur la manière de finir ou de ne pas finir un roman. Les deux chapitres fournissent un témoignage rarement aussi détaillé chez Austen d'une séquence de trois essais de conclusion : premièrement une fin, datée du « 16 juillet 1816 » (fig. 11), elle-même constituée de plusieurs couches de textes, certaines supprimées, même s'il est impossible de déterminer avec certitude quelles suppressions étaient antérieures ou postérieures à l'achèvement provisoire du 16 juillet; une seconde fin, datée de deux jours plus tard « 18 juillet 1816 » (fig. 12), s'appuyant sur et développant des éléments de la première fin; et, à la suite du second finale, encore trois pages de texte, en forme d'explication et de justification, constituant une sorte de nouvelle fin en miniature, accolée comme une pensée après coup, après la conclusion du roman mais destinée à être insérée vers la clôture du chapitre précédent, au chapitre $\mathrm{X}$, et à approfondir, à travers l'acte de rétrospection plus attentif de Wentworth (le personnage masculin principal) ce qui avait été, dans un état antérieur et non révisé du texte, traité trop rapidement à la fois par le personnage et par le narrateur. Il est peut-être étonnant de découvrir que c'est 


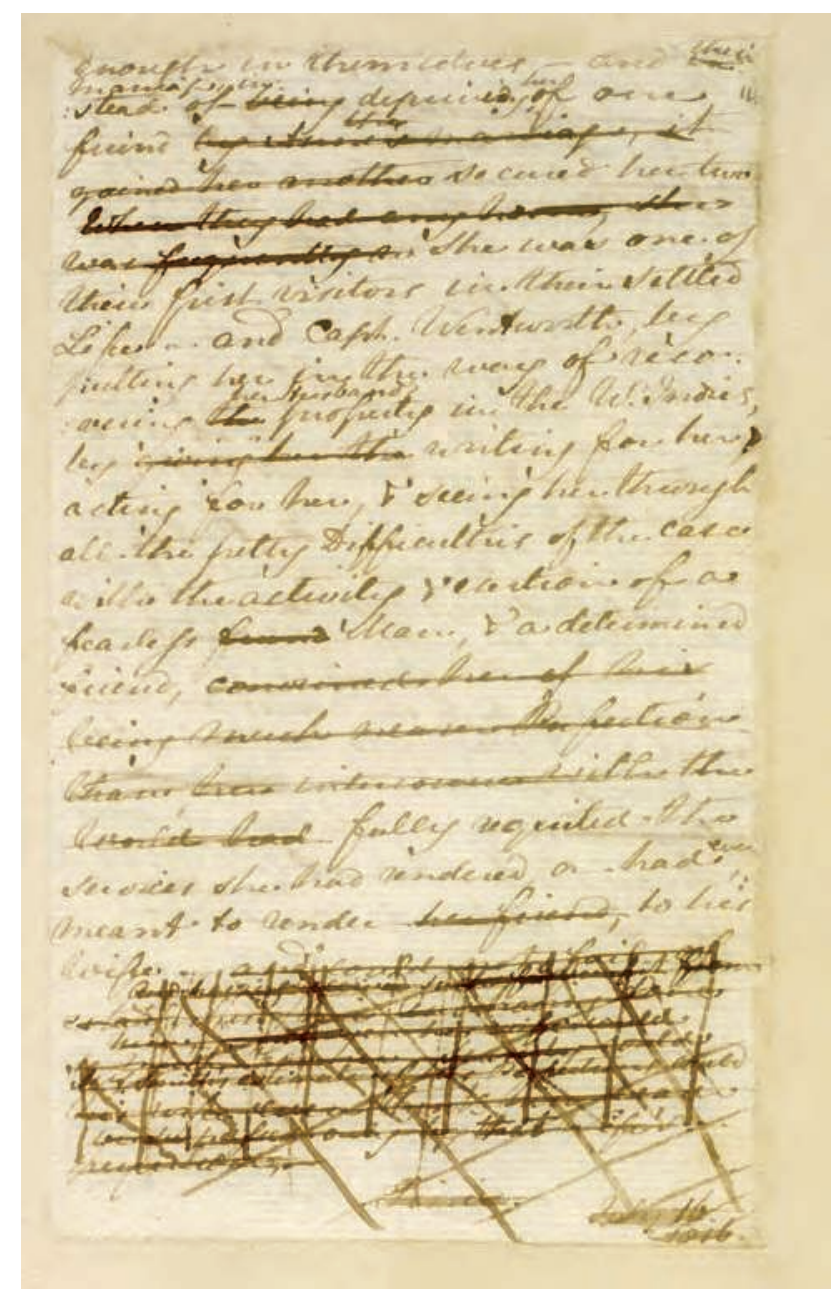

Fig. 11 : Jane Austen, Persuasion, p. 27 (London British Library, Ms. Egerton 3038) (c) London British Library

seulement dans ces trois pages ajoutées très tard (matériellement parlant, la dernière addition de tout le brouillon), qu'Austen fait parler son héroïne Anne Elliot des avantages et des inconvénients éthiques et émotionnels de la «persuasion » - le thème principal du roman.

[«] was not the recollection of what had been — the knowledge of her Influence $-<$ the indelible, immoveable Impression of $>$ of your what Persuasion had once done, was not it all against me ? — « You should have distinguished — replied

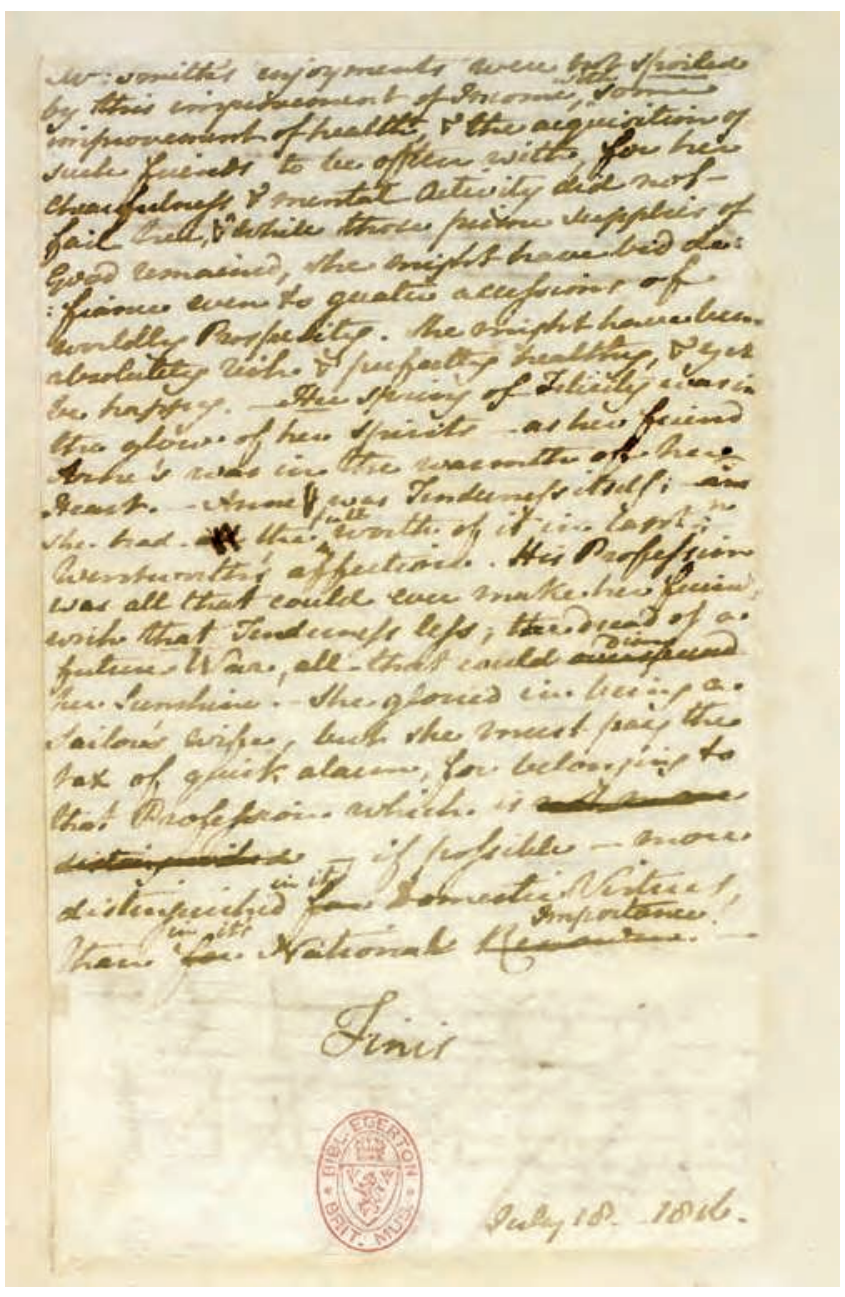

Fig. 12 : Jane Austen, Persuasion, p. 28 (London British Library, Ms. Egerton 3038) (C) London British Library

Anne - You should not have suspected me now; - The case $\wedge^{\text {Was }}$ so different, \& my age so different! — If I was wrong, in yeilding to Persuasion once, remember that it was to Persuasion exerted on the side of safety, not of Risk. [»] (Persuasion, p. 30) (fig. 13).

Malgré la sérialité de leur présentation, il n'y a pas une linéarité simple qui organise ces trois principales phases de révision, ainsi qu'on s'en rend compte quand on les confronte au texte tel qu'il a été remanié dans l'imprimé. 


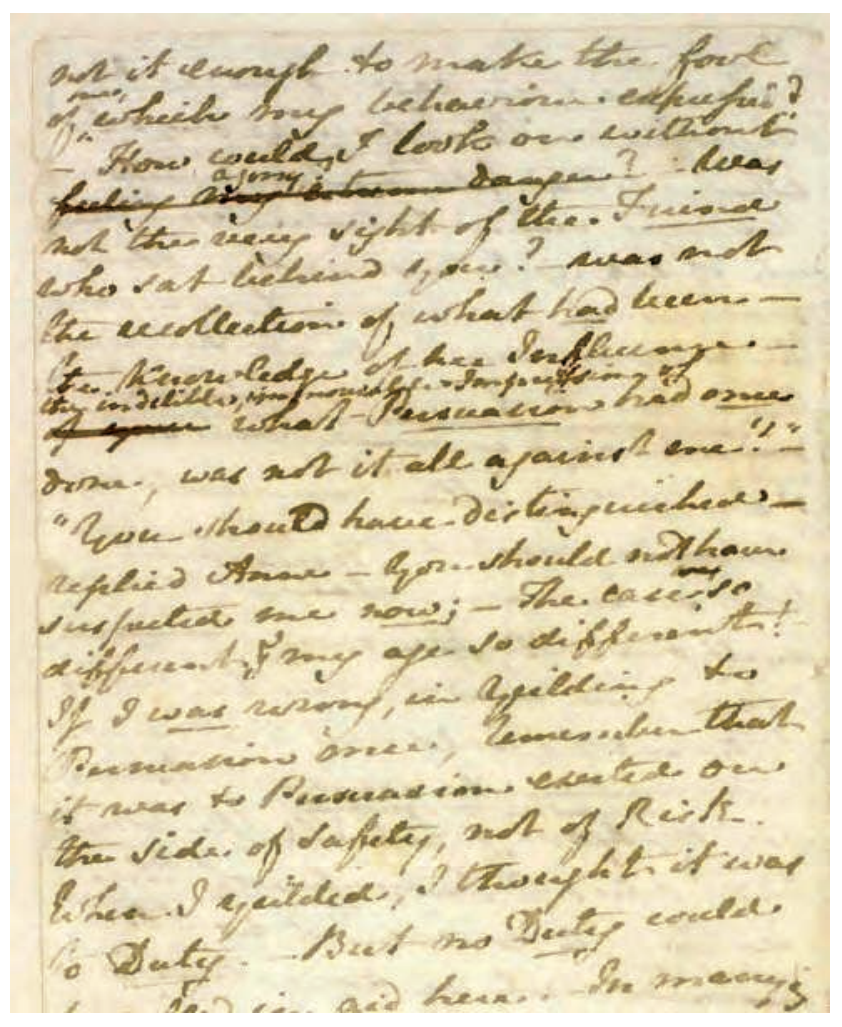

Fig. 13 : Jane Austen, Persuasion, p. 30 (London British Library, Ms. Egerton 3038) (C) London British Library

Au contraire, les révisions dans Persuasion expriment de manière aiguë la frustration que suscite chacun des trois brouillons d'Austen. Ils constituent tous trois des exemples extrêmes de la pratique d'Austen en tant qu'écrivain à processus : deux sont des commencements substantiels sans fin, et le troisième une fin écartée.
Plusieurs passages fortement révisés dans le manuscrit de Persuasion, à quoi s'ajoute la fin nettement différente dans la version imprimée, permettent de comprendre que malgré sa réputation de styliste classique, Austen n'évoluait pas au sein d'un cadre rigide qui lui aurait fait anticiper ses choix ou l'aurait guidée dans son écriture. Manifestement son écriture est rapide, et même maladroite, sans lien avec une intention profonde, non pas seulement ou principalement dans le cadre étroit de la scène qui amène la réconciliation d'Anne Elliot et de Wentworth dans la fin rejetée. Dans la scène telle qu'elle avait été d'abord rédigée chez les Croft, l'intrigue est en fait bien conduite et satisfaisante du point de vue du comique, bien qu'il semble probable que ce soit seulement à ce dernier stade, après coup, qu'Austen ait compris toute l'importance de faire ressortir les diverses implications de la notion de «persuasion ». Avec le brouillon de Persuasion, nous nous heurtons à certains des problèmes les plus ardus posés par les manuscrits d'Austen : à quel point leur apparence et la manière de travailler qu'ils révèlent sont-elles représentatives de sa pratique d'écrivain, y compris pour les romans majeurs dont nous n'avons aucune archive manuscrite ? Les habitudes de la plume sont-elles révélatrices des habitudes de l'esprit et à ce titre autoriseraient-elles des extrapolations au-delà des limites de ce qui est conservé ? Avec Persuasion, seul cas où nous ayons le manuscrit et l'imprimé, le processus de l'écriture devrait élucider le produit fini ; et pourtant ici pas plus qu'ailleurs les morceaux ne coïncident, et de nouveau nous sommes face à l'insuffisance irritante de ce qui demeure.

Traduit de l'anglais par Nathalie Ferrand 
KATHRYN SUTherland est Professeur en bibliographie et critique textuelle à l'Université d'Oxford et Professorial Fellow en anglais à St Anne's College d'Oxford. Ses thèmes de recherche portent sur les effets privés et sociaux ainsi que les valeurs culturelles que l'on attache aux formes des documents, qu'ils soient manuscrits, imprimés ou numériques. Parmi ses publications récentes, on signalera : Jane Austen's Textual Lives: From Aeschylus to Bollywood (2005; en format de poche 2007) ; Transferred Illusions: Digital Technology and the Forms of Print (avec Marilyn Deegan, 2009) ; une édition numérique qui rassemble les manuscrits de fiction de Jane Austen (2010), accessible librement à cette adresse : <www.janeausten.ac.uk> ; et une édition sur papier en quatre volumes de ces manuscrits à paraître en 2015 chez Oxford University Press. Elle prépare actuellement un livre sur les manuscrits littéraires des écrivains.

kathryn.sutherland@st-annes.ox.ac.uk

\section{Résumés \\ Les manuscrits de Jane Austen : sur la page et dans la durée}

En anglais, le terme «manuscrit » est communément utilisé pour désigner deux types de réalité : scripturale et matérielle. Car les manuscrits sont à la fois des inscriptions écrites (des textes) et leurs supports (des objets) ; et lorsque nous parlons d'eux, nous nous référons à ces deux dimensions : le manuscrit littéraire et le manuscrit matériel. Les éditeurs scientifiques négligent parfois la relation qui les unit, mais ce n'est certainement pas le cas de l'écrivain en train de composer ou de transcrire. Les mots sur la page n'offrent à eux seuls qu'un aperçu incomplet du processus de la création ; en tant que support physique du texte écrit, les manuscrits fournissent des indices sur la manière de travailler d'un auteur. En prenant l'exemple de Jane Austen, je propose d'examiner la façon dont ses manuscrits en tant qu'objets éclairent notre compréhension de sa méthode de composition, de sa manière d'écrire.

In English the term "manuscript" is commonly used to cover two kinds of evidence: scribal and physical. That is, manuscripts are both written inscriptions (texts) and their supports (objects); and when we speak of a manuscript we refer to both writing and paper: the literary manuscript and the physical manuscript. Editors sometimes neglect the relationship between the two, but we can be sure that this is not the case for the composing or transcribing author. Words alone offer an incomplete account of the processes of creation; as physical supports for the written text, manuscripts provide clues to an author's way of working. Using the example of Jane Austen I propose to examine how evidence from her manuscripts as objects informs our understanding of her method of composition, her way of writing.

Auf Englisch wird der Begriff „Manuskript“ gewöhnlich verwendet, um zwei Arten von Überliefertem zu bezeichnen: Geschriebenes und dessen materielles Substrat. „Manuskripte“ können wir also sowohl Texte als auch deren physische Träger nennen. Die wissenschaftlichen Herausgeber vernachlässigen manchmal die Beziehung, die zwischen beiden Bedeutungen besteht, was sicherlich nicht auf den Autor selbst zutrifft, der dabei ist, Texte zu verfassen oder zu transkribieren. Wörter, die auf einem Blatt Papier stehen, legen für sich allein genommen nur auf sehr unvollständige Weise Zeugnis vom literarischen Schaffensprozess ab; doch als materielles Substrat und Gerüst eines geschriebenen Texts liefern Manuskripte Hinweise auf die Arbeitsweise eines Autors. Am Beispiel von Jane Austen untersuche ich, wie ihre Manuskripte als Objekte unser Verständnis ihrer Methode der Textkomposition und ihrer Weise des Schreibens erhellen.
In inglese, il termine "manoscritto" è comunemente utilizzato per descrivere due tipi di realtà: una scritturale e l'altra fisica. I manoscritti sono, infatti, sia le iscrizioni scritte (testi) sia i loro supporti (oggetti) e quando parliamo di manoscritti ci riferiamo tanto alla scrittura quanto alla carta, tanto al manoscritto letterario quanto al manoscritto fisico. Gli editori scientifici tralasciano a volte la relazione tra i due, ma di certo non lo fa l'autore che compone o trascrive. Le parole da sole ci offrono un resoconto incompleto del processo creativo; in quanto supporto fisico per il testo scritto, il manoscritto ci fornisce degli indizi sul modo di lavorare dell'autore. Utilizzando l'esempio di Jane Austen, mi propongo di esaminare in che modo i suoi manoscritti, intesi come oggetti, incidono sulla nostra comprensione del suo metodo di composizione, del suo modo di scrivere.

Em inglês, o termo "manuscrito" é comummente usado para designar dois tipos de realidade: escriptural e material. De facto, os manuscritos tanto são as inscrições escritas (os textos) como os seus suportes (objetos); quando falamos deles, referimo-nos a essas duas dimensões de manuscrito literário e de manuscrito físico. Os editores científicos às vezes negligenciam a relação que une essas dimensões, mas esse certamente não é o caso do escritor que compõe ou transcreve. As palavras sobre a página sozinha oferecem uma imagem incompleta do processo de criação; como suporte físico do texto escrito, os manuscritos fornecem pistas sobre como trabalhava um autor. Tomando o exemplo de Jane Austen, proponho-me examinar o modo como os seus manuscritos, enquanto objetos, nos ajudam a compreender o seu método de composição, a sua maneira de escrever.

En inglés, el término "manuscrito" es utilizado comúnmente para designar dos tipos de realidades: escrituraria y material. Puesto que los manuscritos son a la vez inscripciones escritas (textos) y sus soportes (objetos), cuando nos referimos a ellos nos estamos refiriendo a esas dos dimensiones: el manuscrito literario y el manuscrito material. Los editores científicos descuidan a veces las relaciones que las unen, lo que de ninguna manera es el caso del escritor cuando compone o transcribe. Las palabras en la página no pueden brindar más que un vislumbre incompleto del proceso de creación; los manuscritos, en tanto que soporte físico del texto escrito, son reveladores de indicios acerca de la manera de trabajar de un autor. Tomando como ejemplo Jane Austen, me propongo examinar cómo los manuscritos, en tanto que objetos, esclarecen nuestra comprensión de su método de composición, de su forma de escribir. 